\title{
Atomic-scale Observation of Hydroxyapatite Nanoparticle
}

Yi-Sheng Chen ${ }^{1,2^{*}}$, Daniel S. Mosiman ${ }^{1,3}$ Limei Yang $^{1}$, Thi Hanh Pham ${ }^{4}$, Brian Hawkett ${ }^{4}$, Benito J. Mariñas $^{3}$, and Julie M. Cairney ${ }^{1,2}$

1. Australian Centre for Microscopy and Microanalysis, The University of Sydney, Australia.

2. School of Aerospace, Mechanical and Mechatronic Engineering, The University of Sydney, Australia.

3. Department of Civil and Environmental Engineering, University of Illinois at Urbana-Champaign, Illinois, USA.

4. School of Chemistry, The University of Sydney, Australia.

* Corresponding author: yi-sheng.chen@ sydney.edu.au

Fluorosis is a disease known for its major effects in teeth discoloring, arthritic joint pain, and in some cases crippling due to the excess intake of fluoride from drinking water. It is a serious problem for people in low-income, rural areas where excess fluoride is endemic, because they lack access to alternative water sources and cannot afford standard treatment options [1]. This problem can be addressed via using calcium hydroxyapatite (HAP, $\left.\mathrm{Ca}_{5}\left(\mathrm{PO}_{4}\right)_{3} \mathrm{OH}\right)$ nanoparticles (NPs) to exchange the fluoride ion $\left(\mathrm{F}^{-}\right)$in water with the hydroxide ion $\left(\mathrm{OH}^{-}\right)$of HAP to form calcium fluorapatite (FAP, $\left.\mathrm{Ca}_{5}\left(\mathrm{PO}_{4}\right)_{3} \mathrm{~F}\right)$, which is thermodynamically more stable than HAP. This approach has the advantages of cost-effectiveness in mass production, simplicity of operation, and high selectivity for fluoride [1]. However, in practice this technique has only achieved $\mathrm{F}^{-}$uptake at an order of magnitude below its theoretical capacity, and hence, a better understanding of the fundamental $\mathrm{F}^{-}$uptake mechanism(s) of HAP NPs is required for better design/synthesis/manufacturing strategies to produce the future products.

To investigate these mechanism(s), characterization techniques capable of examining individual HAP NPs to concurrently determine their structure and chemical composition are a prerequisite. Transmission electron microscopy (TEM) has been successfully applied to observe HAP NPs at the atomic level and can verify its hexagonal P6/m crystal structure [2]. However, due to the high susceptibility of HAP NPs to electron beam damage, TEM has not yielded convincing data concerning nanometer-level distribution of $\mathrm{F}^{-}$ions on and in individual HAP NPs. To address this, atom probe tomography (APT) is capable because of its advantageous spatial resolution for nanoscale features and chemical precision based upon its time-of-flight spectroscopic configuration. However, progress has been limited by the strict geometry requirement of the APT sample - an ultra-sharp needle with a $50 \mathrm{~nm}$ radius - within which individual HAP NPs (rods averaging $60 \mathrm{~nm}$ long by $20 \mathrm{~nm}$ dia.) must be fixed. To achieve this sample configuration, HAP NPs must be well dispersed on a substrate and encapsulated by a suitable material, followed by focused ion beam (FIB) assisted lift out and needle milling, as demonstrated in [3]. Also, consideration of the compatibility of the encapsulation/substrate materials with HAP to minimize the extent of peak overlaps in APT mass spectra must be considered to acquire high quality data [4].

To address these considerations, a recipe was developed to more evenly disperse HAP NPs in methanol rather than acetone, with no ligand addition to retain the virginity of the material composition, as shown in Figure 1(a) and (b), respectively. HAP encapsulation using a gold substrate and a gold coating was carried out prior to FIB sample preparation, yielding a mass spectrum that contains data without peak overlaps between the encapsulating material and the primary peaks of HAP, as shown in Figure 2(a). The resulting atom probe observation of a HAP NP at atomic scale is shown in Figure 2(b) which suggests the applicability of the proposed protocol to provide insights into the fluoride uptake mechanism(s) of HAP NPs. [5] 


\section{References:}

[1] J.K.Fawell et al. 'Fluoride in drinking-water', WHO drinking water quality series, (2006, London, IWA Publishing)

[2] L. Bertinetti, et al., Journal of Physical Chemistry C 111 (2017), p.4027-4035

[3] P. Felfer, et al., Angewandte Chemie International Edition 53 (2014), p.11190-11193.

[4] L. M. Gordon, L. Tran, and D. Joester, ACS Nano 6 (2012), p.10667-10675

[5] The authors acknowledge funding from the Australian Research Council (DP160101713) as well as the support of research facilities from Microscopy Australia, Sydney Microscopy and Microanalysis, and Key Centre for Polymers and Colloids. D. S. M. thanks the U.S. National Science Foundation Graduate Research Fellowship and the American Water Works Association Larson Aquatic Research Support Doctoral Scholarship. Y.-S. C. is grateful for the University of Sydney Fellowship as well as the support from his family.

(a)

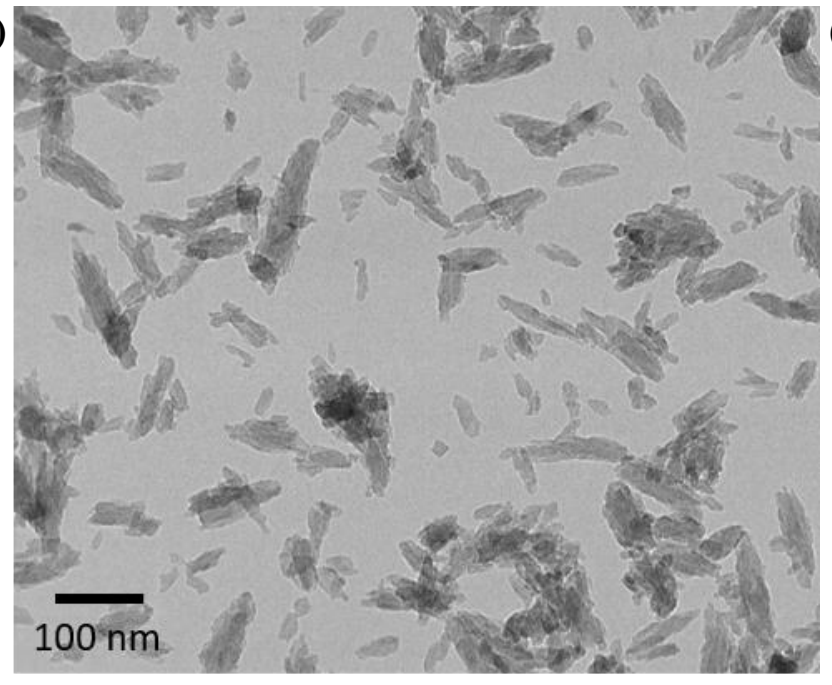

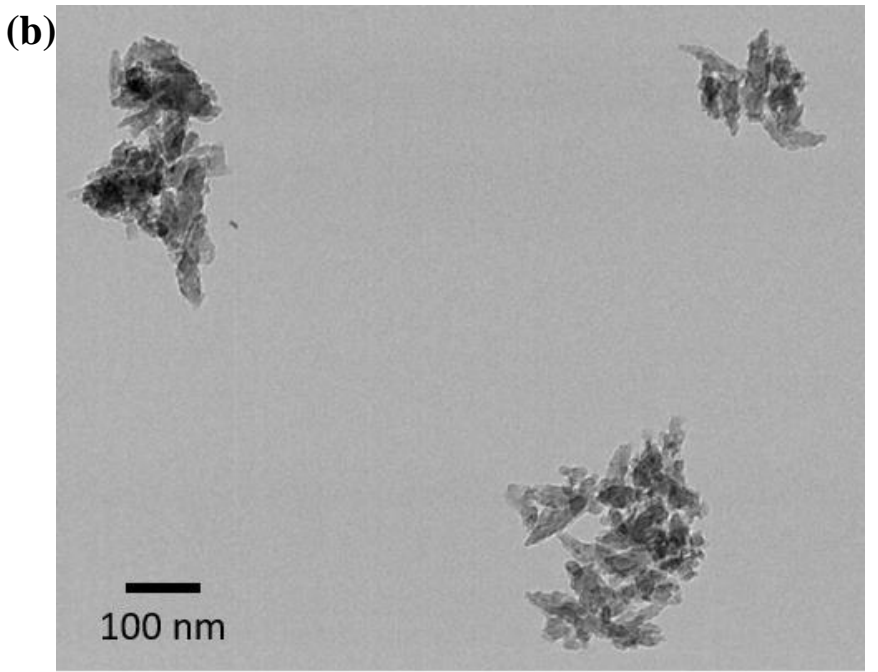

Figure 1. TEM images of the HAP particles dispersed in (a) methanol and (b) acetone where the former shows improved dispersion of particles
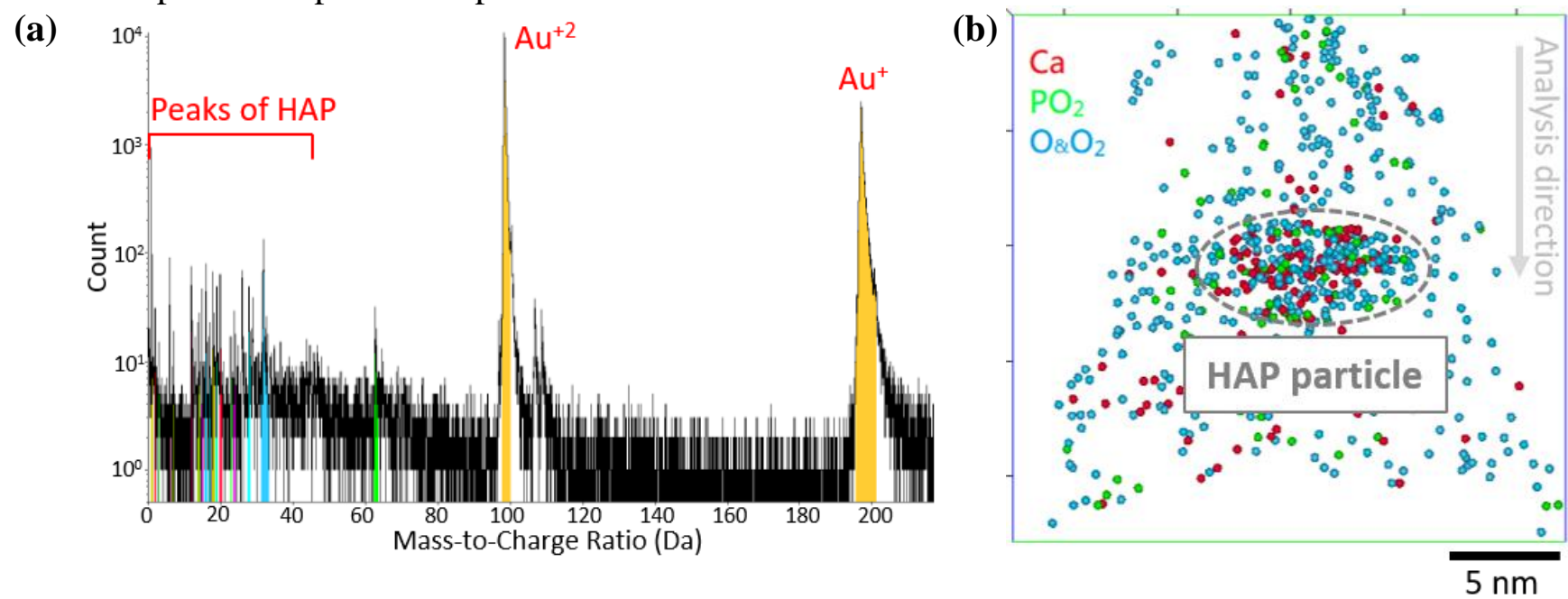

Figure 2. (a) Mass spectrum of an atom probe specimen of gold incorporating HAP a particle. (b) 5-nm slice of the $3 \mathrm{D}$ atom map of HAP related ions $\left(\mathrm{Ca}, \mathrm{PO}_{2}, \mathrm{O}\right.$ and $\left.\mathrm{O}_{2}\right)$ showing the presence of HAP particle within the dataset. 